\title{
Deafness, Autosomal Recessive 4, with Enlarged Vestibular Aqueduct
}

National Cancer Institute

\section{Source}

National Cancer Institute. Deafness, Autosomal Recessive 4, with Enlarged Vestibular

Aqueduct. NCI Thesaurus. Code C158787.

An autosomal recessive condition caused by mutation(s) in one of several genes, most often SLC26A4 encoding pendrin. It is characterized by hearing loss and enlargement of the vestibular aqueduct. Mutation(s) in the SLC26A4 gene also cause Pendred syndrome. 